\title{
A Novel Method of Radar Emitter Identification Based on the Coherent Feature
}

\author{
Jian Xue ${ }^{1,2}$, Lan Tang ${ }^{1, *(\mathbb{D})}$, Xinggan Zhang ${ }^{1}$ and Lin Jin ${ }^{1,2}$ \\ 1 School of Electronic Science and Engineering, Nanjing University, Nanjing 210023, China; \\ dg1523030@smail.nju.edu.cn (J.X.); zhxg@nju.edu.cn (X.Z.); jinl@smail.nju.edu.cn (L.J.) \\ 2 Nanjing Research Institute of Electronics Technology, Nanjing 210039, China \\ * Correspondence: tanglan@nju.edu.cn
}

Received: 15 June 2020; Accepted: 21 July 2020; Published: 30 July 2020

\begin{abstract}
To deal with the problem of reliability degradation of radar emitter identification (REID) based on the traditional five parameters in a complex electromagnetic environment, a new feature extraction method based on the autocorrelation function of coherent signals, which makes full use of the coherent characteristic of modern radar emitters, is proposed in this paper. The main idea of this paper is utilizing the instantaneous autocorrelation function to obtain the correlation results of coherent and noncoherent signals. To this end, a new feature parameter, named the ratio of the secondary peak value to the main peak value (SMR), is defined to describe the difference of correlation results between coherent and noncoherent signals. Through simulation analysis, the feasibility of using SMR as the coherent feature for REID is verified. In order to evaluate the effectiveness of the coherent feature, an analytical hierarchy process (AHP) was introduced to compare the comprehensive performance of the coherent feature and the existing parameters, and then convolution neural network $(\mathrm{CNN})$ and support vector machine (SVM) were selected as the classifier to check the recognition capability of the proposed feature. Simulation results show that the proposed feature can not only be used as a new feature for REID but can also be used as a supplement to existing feature parameters to improve the accuracy of REID as it is more insensitive to the signal-to-noise ratio (SNR) and signal modulation type changes.
\end{abstract}

Keywords: radar emitter identification; coherent feature; instantaneous autocorrelation function; SMR; analytical hierarchy process (AHP)

\section{Introduction}

In the signal processing of a radar reconnaissance system, radar emitter identification (REID) is one of the crucial aspects [1]. Recognition performance has become an important symbol to measure the technology levels of radar countermeasure equipment [2,3]. With the wide application of modern radar and the degree increase of electronic radar countermeasures (there are all kinds of jamming), the electromagnetic environment is becoming more and more complex. The feature parameters, such as radio frequency (RF), pulse repeat interval (PRI), pulse wide (PW), angle of arrival (AOA), and pulse amplitude (PA) of radar emitter signals measured by reconnaissance equipment, are always illegible and uncertain, which bring out two problems in REID. One is that recognition performance may be decreased because of the uncertainty of the emitter signals. The other is the inability to update the emitter databases because there are no credible feature parameters.

With the development of information technology, domestic and foreign scholars have done a lot of indepth and systematic studies in REID. At present, the research results can be divided into two categories. One is the intelligent classifier design, which can process the feature parameter uncertainties of the measured emitter signals and classify them correctly. Many conventional recognition classifiers 
have been successfully applied, such as fuzzy [4], Bayesian [5], k-nearest neighbor [6], support vector machine [7], neural network [8-10], and so on. They can solve the problem caused by measurement uncertainty or error to a certain extent. However, when there are no credible feature parameters for the classifier, these classification methods are also helpless. The other category is trying to extract a new feature parameter to make up for the shortcomings of the existing five feature parameters in emitter recognition. Presently, many studies are concerned with the existence of modulation on radar pulses (MOPs), such as entropy [11], resemblance coefficient [12,13], wavelet gray matrix [14-16], and so on. However, when the two radars are deployed in close proximity and their signal parameters and modulation types are the same, these recognition methods based on the MOP feature are almost invalid. In this paper, the primary focus is on extracting a new feature parameter.

In recent years, with the rapid development of modern radar technology, most radars in use are of the coherent system $[17,18]$. The coherent system denotes that the initial phases of the transmitting signals from a coherent radar are continuous and stable, while those from a noncoherent radar are random. The correlation function [19-21] describes the correlation degree among the instantaneous values of random sample functions at different times, and coherence represents the phase relationship between the adjacent pulses, which is correlated and continuous. Therefore, the instantaneous autocorrelation function is introduced to distinguish the coherent characteristics of these pulses.

In this paper, the feasibility of the coherent feature as a feature parameter for REID is demonstrated firstly by theoretical analysis. Then, the instantaneous autocorrelation function is taken to obtain the correlation differences between the coherent and noncoherent signals, and a new feature parameter named the ratio of the secondary peak value to the main peak value (SMR) is defined as the coherent feature to describe these differences. Through the simulation analysis, the simulation result shows that the new feature parameter (SMR) is not sensitive to signal-to-noise ratio (SNR) and signal type changes. To evaluate the recognition performance of the coherent feature, an analytical hierarchy process (AHP) is introduced, which makes a comprehensive evaluation from the three aspects of reliability, accuracy, and robustness of SMR and the existing parameters. Then, a sketch map of REID is constructed and applied in simulation experiments, according to the comprehensively evaluated results. Furthermore, support vector machine (SVM) and convolution neural network (CNN) are introduced as the classifiers to check the recognition capability of the proposed feature compared with the typical five parameters.

The remainder of this paper is organized as follows. In Section 2, the model of received signals from a coherent emitter is given, and the feasibility of the coherent feature as the REID feature is proved by theoretical analysis. In Section 3, simulations by an instantaneous autocorrelation function on the coherent and noncoherent signals are presented. Through simulation analysis, the discrimination model and the discrimination threshold of coherent characteristics are introduced to distinguish correlation results of the coherent and noncoherent signals. In Section 4, three experiments are performed to demonstrate the identification capability of the proposed coherent feature. Conclusions are made in Section 5 .

\section{Analysis of Signal Coherent Characteristics}

As we all know, the premise of emitter identification is to classify the received pulses from the same radar emitter into one class by some criteria. In view of the stable phase relationship between the signals transmitted from the coherent radar, the purpose of analyzing the signal's coherent characteristics is to find out the relationship of the initial phase among the received pulse train, so as to use this feature to distinguish the coherent and noncoherent signals for REID.

In this section, the received signal model is given. Then, the phase relationship between the received pulse trains is analyzed to validate the feasibility of using the coherent feature as the recognition feature. The relationship between the reconnaissance equipment and the target radar in a radar reconnaissance system is shown in Figure 1; this figure is only a sketch map between the radar emitter and the receiving equipment. The key point is to derive the relationship between the initial 
phases of the pulse train from the same radar emitter. Therefore, multipath and other jammings in the actual environment are not listed in this sketch map.

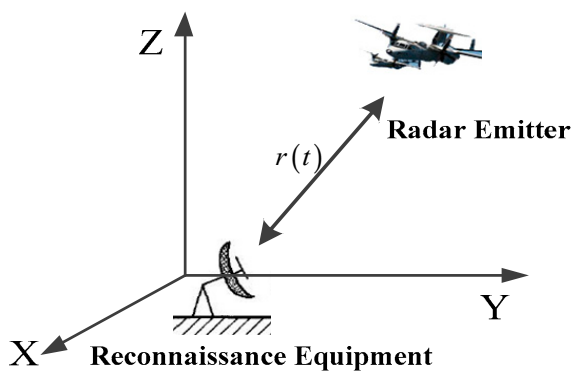

Figure 1. Relationship between the reconnaissance equipment and the radar emitter.

In Figure $1, r(t)$ denotes the relative distance between the receiving equipment and the target radar. Supposing $x(t)$ is the signal transmitted from the target radar and $s(t)$ is the received signal, the relationship between $x(t)$ and $s(t)$ can be shown, as below.

$$
\left\{\begin{array}{l}
s(t)=u x(t-\Delta)+v(t) \\
\Delta \approx r(t) / c
\end{array}\right.
$$

where $u$ denotes the attenuation coefficient of signal amplitude, $c$ is the signal transmission speed, $v(t)$ is the noise.

$x(t)$ is defined as the transmitting signal from the radar emitter; it can be expressed as follows:

$$
x(t)=A \exp \left[\mathrm{j}\left(2 \pi f_{c} t+\phi_{0}\right)\right]
$$

where $A, f_{c}$, and $\phi_{0}$ denote the amplitude, frequency, and initial phase of the transmitting signal. Without considering signal attenuation, the received signal $s(t)$ can be expressed as follows:

$$
s(t)=A \exp \left\{\mathrm{j}\left[2 \pi f_{c}(t-r(t) / c)+\phi_{0}\right]\right\}+v(t)
$$

When $t=t_{0}$, expanding $r(t)$ according to Taylor's formula,

$$
r(t)=r\left(t_{0}\right)+r^{\prime}\left(t_{0}\right)\left(t-t_{0}\right)+\frac{1}{2 !} r^{\prime \prime}\left(t_{0}\right)\left(t-t_{0}\right)^{2}+\cdots+\frac{1}{n !} r^{(n)}\left(t_{0}\right)\left(t-t_{0}\right)^{n}+\cdots
$$

Substitute $r(t)$ into $s(t)$, then the received signal $s(t)$ can also be expressed as below.

$$
s(t)=A \cdot \exp \left\{\mathrm{j}\left\{2 \pi f_{\mathcal{c}}\left\{t-\frac{1}{c}\left[r\left(t_{0}\right)+r^{\prime}\left(t_{0}\right)\left(t-t_{0}\right)+\frac{1}{2} r^{\prime \prime}\left(t_{0}\right)\left(t-t_{0}\right)^{2}+\cdots\right]\right\}+\phi_{0}\right\}\right\}+v(t)
$$

The phase function $\varphi(t)$ of received signal $s(t)$ is as follows:

$$
\varphi(t)=2 \pi f_{c} t-2 \pi\left[\frac{r\left(t_{0}\right)}{\lambda}+\frac{r^{\prime}\left(t_{0}\right)}{\lambda}\left(t-t_{0}\right)+\frac{1}{2} \frac{r^{\prime \prime}\left(t_{0}\right)}{\lambda}\left(t-t_{0}\right)^{2}+\cdots\right]+\phi_{0}
$$

Due to the relative motion between the target emitter and the receiving equipment, which is reflected in the Doppler effect $\left(f_{d}\right)[22], \varphi(t)$ can be expressed as below.

$$
\varphi(t)=\varphi_{0}+2 \pi\left[\left(f_{c}+f_{d}\right)\left(t-t_{0}\right)+\frac{1}{2} f_{d}{ }^{\prime}\left(t_{0}\right)\left(t-t_{0}\right)^{2}+\cdots+\frac{1}{n !} f_{d}^{(n)}\left(t_{0}\right)\left(t-t_{0}\right)^{n}\right]
$$

where $\varphi_{0}\left(\varphi_{0}=\phi_{0}+2 \pi f_{\mathcal{c}}\left[t_{0}-r\left(t_{0}\right) / c\right]\right)$ denotes the initial phase of the received signal at the time $t_{0}$. It is to be noted that if the radar emitter is stationary, there is no Doppler effect $\left(f_{d}=0\right)$. 
Then, using the second-order approximation to describe $r(t), \varphi(t)$ can be further simplified as below.

$$
\varphi(t) \approx \varphi_{0}+2 \pi\left(f_{c}+f_{d}\right)\left(t-t_{0}\right)+\pi f_{d}^{\prime}\left(t_{0}\right)\left(t-t_{0}\right)^{2}
$$

When $t_{0}=0$, it is assumed that the Doppler change rate $\left(f_{d}{ }^{\prime}\left(t_{0}\right)\right)$ can be ignored in a very short observation time, so the phase function $\varphi(t)$ can be expressed as below.

$$
\varphi(t)=\varphi_{0}+2 \pi\left(f_{c}+f_{d}\right) t
$$

According to Formula (9), the received pulse train can be expressed as follows.

$$
s(t)=\sum_{p=1}^{N} A_{p} \cdot \operatorname{rect}\left(\frac{t-T_{p}}{\tau}\right) \exp \left\{\mathrm{j}\left[2 \pi\left(f_{c p}+f_{d}\right) t+\varphi_{0}\right]\right\}+v(t)
$$

where $A_{p}, T_{p}, f_{c p}$ represent the PA, time of arrival (TOA), RF of the $p$ th pulse in a pulse train, rect( $(\cdot)$ denotes the rectangular window function, $N$ is the number of the received pulses. After passing through the mixer, $s(t)$ can be expressed as below.

$$
s(t)=\sum_{p=1}^{N} A_{p} \cdot \operatorname{rect}\left(\frac{t-T_{p}}{\tau}\right) \exp \left\{j\left[2 \pi f_{p} t+\varphi_{0}\right]\right\}+v(t)
$$

where $f_{p}$ denotes the center frequency (CF) of the $p$ th pulse in a pulse train, $f_{p}=f_{c p}+f_{d}-f_{L},\left(f_{d}<<f_{c p}\right)$, $f_{L}$ denotes the local frequency.

From the analysis above, it can be seen that there is a stable and continuous relationship among the initial phases of the received pulse train transmitted from a coherent radar. Therefore, we can use the coherent feature as the recognition feature for REID.

\section{Coherent Feature Extraction and Evaluation}

In a complex electromagnetic environment, if the coherent feature is extracted from the time domain waveform of the radar signal directly, it is easily affected by noise [23]. The autocorrelation function reflects the correlation degree between the two signals. Considering that the correlation of noise is poor, while the correlation of signals is strong and the noise is not related to the signal, instantaneous autocorrelation processing can strengthen the signal and weaken the noise; therefore, the instantaneous autocorrelation function can be used to extract the coherent feature [24].

Supposing $s(n)$ denotes the received signal, its instantaneous autocorrelation function can be expressed as follows.

$$
\begin{gathered}
Y(n, m)=s(n) \times s^{*}(n+m) \quad m=0, \pm 1, \cdots \\
Y(n)=\sum_{m=-\infty}^{+\infty} Y(n, m)=\sum_{m=-\infty}^{+\infty} s(n) \times s^{*}(n+m)
\end{gathered}
$$

Where $s^{*}(\cdot)$ is the conjugate function of $s(\cdot)$.

From Formula (13), it can be seen that the biggest advantage of instantaneous autocorrelation is that there is no time integration. In other words, the instantaneous information of correlation processing is well preserved.

\subsection{Simulation Analysis}

In order to analyze the difference of coherent characteristics between the coherent and noncoherent pulse trains, three simulation experiments were performed based on time-frequency correlation. We took two sets of pulse trains: the first one was a coherent pulse train and the second a noncoherent 
pulse train. The signal type and the parameters of both pulse trains were the same. It is noteworthy that the number of pulses used for emitter identification must be greater than or equal to 4 .

Simulation Experiment 1: Signal modulation type is continuous wave (CW), CF is $30 \mathrm{MHz}$, sampling frequency (Fs) is $100 \mathrm{MHz}$, PRI is $60 \mu \mathrm{s}$, PW is $10 \mu \mathrm{s}$, pulse number is 10. Autocorrelation results of the two groups, without noise, are shown in Figure 2.

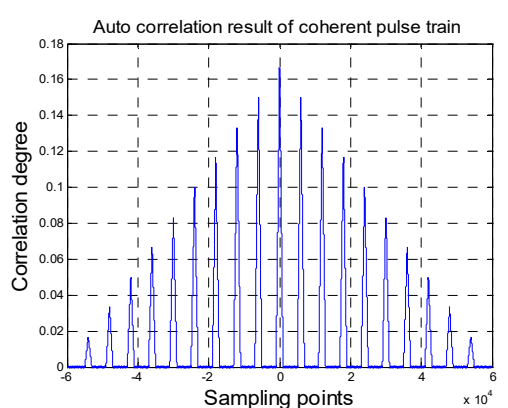

(a) Coherent pulse train.

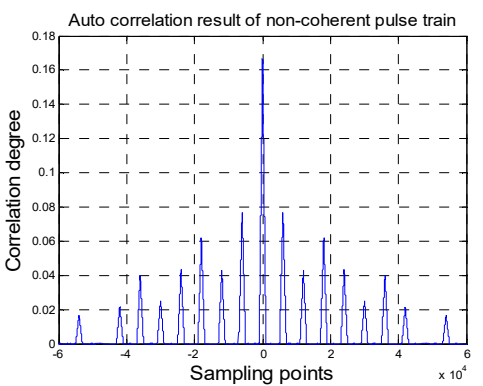

(b) Noncoherent pulse train.

Figure 2. Autocorrelation result of coherent and noncoherent pulse trains; signal type is continuous wave $(\mathrm{CW})$.

Simulation Experiment 2: Signal modulation type is linear frequency modulation (LFM), CF is $30 \mathrm{MHz}$, bandwidth (BW) is $10 \mathrm{MHz}$, Fs is $100 \mathrm{MHz}$, PRI is $150 \mu \mathrm{s}, \mathrm{PW}$ is $15 \mu \mathrm{s}$, pulse number is 8 . Autocorrelation results of the two groups, without noise, are shown in Figure 3.

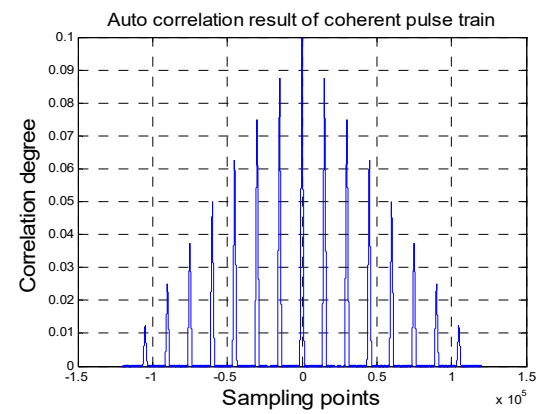

(a) Coherent pulse train.

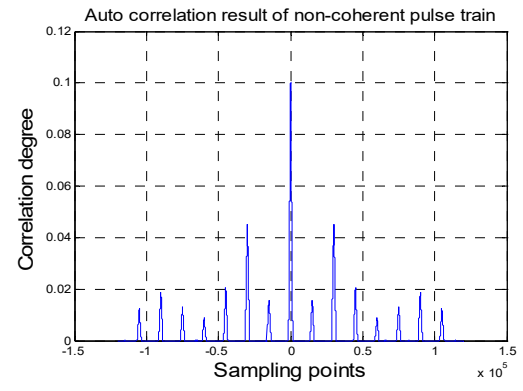

(b) Noncoherent pulse train.

Figure 3. Autocorrelation result of coherent and noncoherent pulse trains; signal type is linear frequency modulation (LFM).

Simulation Experiment 3: Signal modulation type is nonlinear frequency modulation (NLFM), $\mathrm{CF}$ is $100 \mathrm{MHz}, \mathrm{BW}$ is $20 \mathrm{MHz}, \mathrm{Fs}$ is $200 \mathrm{MHz}$, PRI is $50 \mu \mathrm{s}$, PW is $5 \mu \mathrm{s}$, pulse number is 15 . Autocorrelation results of the two groups, without noise, are shown in Figure 4. 


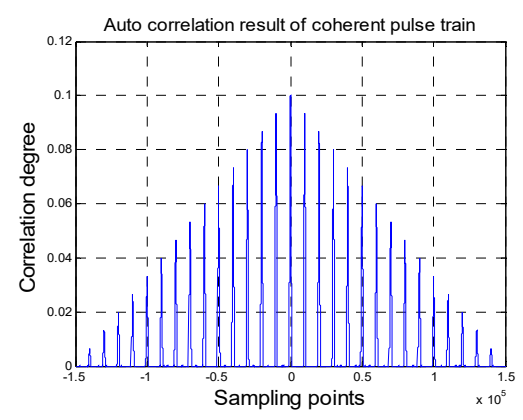

(a) Coherent pulse train.

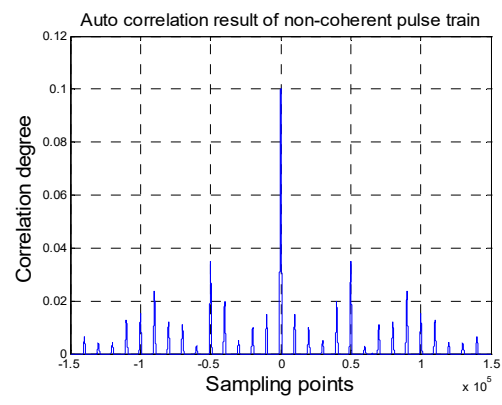

(b) Noncoherent pulse train.

Figure 4. Autocorrelation result of coherent and noncoherent pulse trains; signal type is nonlinear frequency modulation (NLFM).

As shown in Figures 2-4, it can be seen that the three experiments of different modulation types have similar rules: the correlation degree between the coherent and noncoherent pulse trains is almost the same near the zero point, while outside the zero point, the correlation of the coherent signals is much better than that of the noncoherent signals. With increasing time delay, the correlation of the coherent signals decreases periodically and steadily, while it changes irregularly in the noncoherent signals. This is due to the strong correlation and continuity of the phase information of the coherent pulse train, while that of the noncoherent pulse train is random. Therefore, we can use this difference to distinguish the coherent and noncoherent signals. The next task is to define a feature parameter to describe this difference.

\subsection{The Model of Coherent Feature Extraction}

According to the simulation analysis above, define the maximum peak at the zero point as the main peak. Except for the maximum peak, the maximum of the remaining peaks is defined as the secondary peak. The discrimination model of coherent characteristics can be named as the ratio of the secondary peak value to the main peak value (SMR). The model parameter is given as below.

$$
S M R=\frac{\max [F(n)]}{F(1)}, \quad n>1
$$

where $F(1)$ is the main peak value at the zero point.

In terms of the conditions of Experiments 1-3 to calculate the SMR of different signal types, take the average of 100 times of calculation results. SMRs are computed without noise in Table 1.

Table 1. Results of the secondary peak value to the main peak value (SMR) with different signal types.

\begin{tabular}{ccccccc}
\hline \multirow{2}{*}{ Signal Type } & \multicolumn{3}{c}{ Coherent Pulse Train } & \multicolumn{3}{c}{ Noncoherent Pulse Train } \\
\cline { 2 - 7 } & CW & LFM & NLFM & CW & LFM & NLFM \\
\hline SMR & 0.904 & 0.889 & 0.928 & 0.463 & 0.408 & 0.392 \\
\hline
\end{tabular}

As shown in Table 1, it can be seen that the SMRs of the coherent pulse train are close to 1, while the SMRs of the noncoherent pulse train are much smaller than 1 .

In order to verify the rationality of the discrimination threshold of SMR in different SNRs, according to the conditions of Experiments 1 to 3, SMRs of different signal types are computed based on the Monte-Carlo for 100 times in different SNRs. The results are shown in Figure 5. 


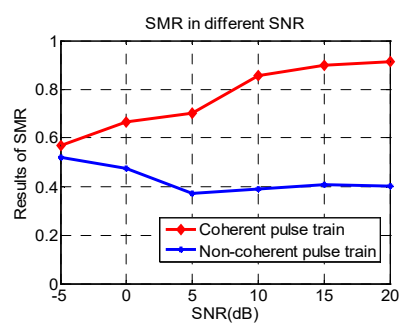

(a) Signal type is CW.

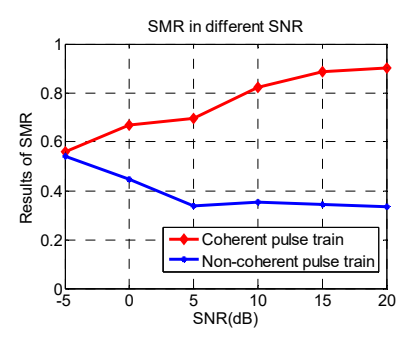

(b) Signal type is LFM.

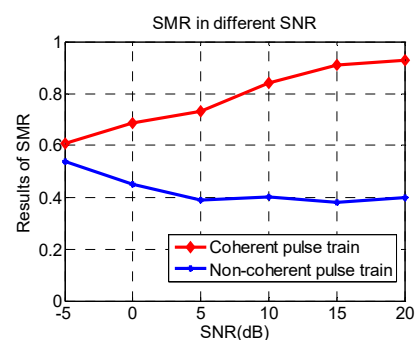

(c) Signal type is NLFM.

Figure 5. SMRs (the ratios of the secondary peak value to the main peak value) of different signal types in different signal-to-noise ratios (SNRs).

As can be seen in Figure 5, when SNR $=-5 \mathrm{~dB}$, SMRs of different signal types are overlapped as SMR cannot distinguish the difference very well. When SNR $\geq 0 \mathrm{~dB}$, the difference in SMRs between the coherent and noncoherent pulse trains is obvious. This is due to the instantaneous autocorrelation processing, which can weaken the noise and strengthen the signal. To a certain extent, SNR of the signal has been improved. From the simulation analysis above, we can conclude that SMR can be used as a feature parameter for REID, and it is insensitive to SNR and signal type changes.

Furthermore, we can see when SNR $\geq-5 \mathrm{~dB}$, SMR of the coherent pulse train is always above 0.6, while SMR of the noncoherent pulse train is always below 0.6. Additionally, each signal type has the same rule. Therefore, we can set the discrimination threshold at 0.6. When SMR $>0.6$, the pulse train is coherent, otherwise the pulse train is noncoherent.

\subsection{Coherent Feature Evaluation}

To evaluate the comprehensive identification performance of SMR and the typical five feature parameters, AHP [25] is introduced in this paper. AHP is a systematic method that takes a complex multiobjective decision-making problem as a system and decomposes the objective into several levels, namely, multiobjective, multicriteria, and multiattribute. Through quantitative and qualitative analyses, the single ranking and total ranking of the levels are calculated as a systematic method of objective and optimized decision-making.

Evaluation steps based on AHP are given as below.

Step 1: Establish a model of the evaluation system

To establish a suitable feature evaluation system as the basis of efficient evaluation, the feature evaluation system needs to follow certain evaluation criteria, such as purposefulness, a scientific nature, and systematization. In this paper, the feature evaluation system is designed as a three-layer structure, namely, a target layer, an index layer, and a scheme layer. The target layer denotes the radar emitter identification. In the index layer, three metrics—reliability, accuracy, and robustness-are adopted to evaluate the performance of the parameters. The scheme layer is the parameters to be evaluated, which are CF, PRI, PW, AOA, PA, and SMR. The model of the evaluation system is shown in Figure 6.

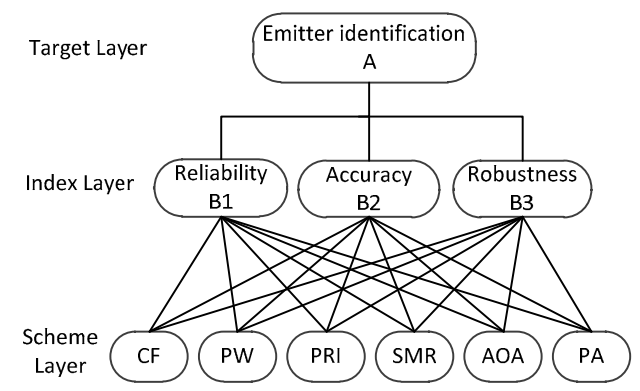

Figure 6. Evaluation system model. 
Step 2: Construct the judgment matrix.

According to the Saaty relative importance hierarchy table [26], reliability, accuracy, and robustness in the index layer are taken to make a comprehensive evaluation of the evaluated feature. The judgment matrix is constructed in terms of these three indexes, which are compared with each other by the expert's scoring. Judgment matrix $A$ is computed below.

$$
A=\left[a_{i j}\right]_{3 \times 3}=\left[\frac{\alpha_{i}}{\alpha_{j}}\right]_{3 \times 3}=\left[\begin{array}{ccc}
1 & 3 & 8 \\
1 / 3 & 1 & 6 \\
1 / 8 & 1 / 6 & 1
\end{array}\right]
$$

where $a_{13}=a_{1} / a_{3}=8$ denotes that the influence of reliability on the evaluation system is much higher than that of robustness for radar emitter identification. The definition of the other elements in matrix $A$ can be deduced in the same way.

In the same way, judgment matrices $B_{1}, B_{2}, B_{3}$ can be obtained below.

$$
\begin{aligned}
& \boldsymbol{B}_{1}=\left[\begin{array}{cccccc}
1 & 1 / 5 & 1 / 7 & 1 / 9 & 3 & 1 / 7 \\
5 & 1 & 1 / 3 & 1 / 5 & 7 & 1 / 3 \\
7 & 3 & 1 & 1 / 3 & 7 & 1 \\
9 & 5 & 3 & 1 & 9 & 3 \\
1 / 3 & 1 / 7 & 1 / 7 & 1 / 9 & 1 & 1 / 7 \\
7 & 3 & 1 & 1 / 3 & 7 & 1
\end{array}\right] \\
& \boldsymbol{B}_{2}=\left[\begin{array}{cccccc}
1 & 3 & 4 & 4 & 5 & 7 \\
1 / 3 & 1 & 3 & 3 & 4 & 6 \\
1 / 4 & 1 / 3 & 1 & 1 & 3 & 5 \\
1 / 4 & 1 / 3 & 1 & 1 & 3 & 5 \\
1 / 5 & 1 / 4 & 1 / 3 & 1 / 3 & 1 & 3 \\
1 / 7 & 1 / 6 & 1 / 5 & 1 / 5 & 1 / 3 & 1
\end{array}\right] \\
& \boldsymbol{B}_{3}=\left[\begin{array}{cccccc}
1 & 3 & 3 & 3 & 5 & 9 \\
1 / 3 & 1 & 1 & 1 & 3 & 7 \\
1 / 3 & 1 & 1 & 1 & 3 & 7 \\
1 / 3 & 1 & 1 & 1 & 3 & 7 \\
1 / 5 & 1 / 3 & 1 / 3 & 1 / 3 & 1 & 5 \\
1 / 9 & 1 / 7 & 1 / 7 & 1 / 7 & 1 / 5 & 1
\end{array}\right]
\end{aligned}
$$

In judgment matrix $B_{1}, \beta_{1} / \beta_{2}=1 / 5$ denotes the reliability of $C F$ is weaker than that of PW, $\beta_{1} / \beta_{5}=3$ denotes the reliability of $C F$ is better than that of AOA. The definition of other elements in matrices $B_{1}, B_{2}, B_{3}$ can be deduced in this way.

Step 3: Calculate the weight vector of judgment matrices $A, B_{1}, B_{2}, B_{3}$ and check their consistency.

In this step, we need to introduce three definitions [27]: compatibility index (CI), random consistency index $(R I)$, and compatibility ratio $(C R)$. The mathematical expression of $C I$ and $C R$ are given below.

$$
\begin{gathered}
C I=\frac{\left(\lambda_{\max }-n\right)}{(n-1)} \\
C R=\frac{C I}{R I}
\end{gathered}
$$

In the formulas mentioned above, $n$ denotes the order of matrix $A, \lambda_{\max }$ is the maximum eigenvalue of the judgment matrix $A$. When $n=3, R I=0.58 ; n=4, R I=0.96 ; n=5, R I=1.12 ; n=6, R I=1.24$ [28].

The rules for consistency testing are as follows: when $C R<0.1$, it is considered that the inconsistency scale of judgment matrix $A$ is in the allowable range and can be accepted. If $C R \geq 0.1$, 
go back to Step 2 to adjust judgment matrix $A$ again, then calculate and check the consistency until it meets the consistency condition $(C R<0.1)$.

According to the Formulas (19) and (20) and judgment matrix $A$, the maximum eigenvalue and the normalized eigenvector of judgment matrix $A$ can be computed as below.

$$
\lambda_{\max }=3.0735, \omega_{A}=\left[a_{1}, a_{2}, a_{3}\right]=[0.65270 .28510 .0623], C I_{A}=0.0367, C R_{A}=0.0633
$$

where $C R_{A}<0.1$, it denotes that matrix $A$ meets the requirement of consistency testing.

The maximum eigenvalues $\lambda_{B_{1}}, \lambda_{B_{2}}, \lambda_{B_{3}}$ and the normalized eigenvectors $\omega_{B_{1}}=\left[b_{11}, b_{12}, b_{13}, b_{14}, b_{15}, b_{16}\right], \omega_{B_{1}}=\left[b_{21}, b_{22}, b_{23}, b_{24}, b_{25}, b_{26}\right], \omega_{B_{3}}=\left[b_{31}, b_{32}, b_{33}, b_{34}, b_{35}, b_{36}\right]$ of matrices $B_{1}, B_{2}, B_{3}$ can be computed in the same way. The computation results are shown in Table 2 .

Table 2. Results of consistency testing of matrices $B_{1}, B_{2}, B_{3}$.

\begin{tabular}{ccccccccccc}
\hline $\boldsymbol{k}$ & $\boldsymbol{\lambda}_{\boldsymbol{k}}$ & $\boldsymbol{b}_{\boldsymbol{k} \boldsymbol{1}}$ & $\boldsymbol{b}_{\boldsymbol{k} 2}$ & $\boldsymbol{b}_{\boldsymbol{k} 3}$ & $\boldsymbol{b}_{\boldsymbol{k} 4}$ & $\boldsymbol{b}_{\boldsymbol{k} 5}$ & $\boldsymbol{b}_{\boldsymbol{k} 6}$ & $\boldsymbol{C I}_{\boldsymbol{k}}$ & $\boldsymbol{R I}_{\boldsymbol{k}}$ & $\boldsymbol{C R}_{\boldsymbol{k}}$ \\
\hline 1 & 6.4084 & 0.0371 & 0.1068 & 0.2027 & 0.4261 & 0.0246 & 0.2027 & 0.0817 & 1.24 & 0.0659 \\
2 & 6.3257 & 0.4194 & 0.2447 & 0.1218 & 0.1664 & 0.0604 & 0.0318 & 0.0651 & 1.24 & 0.0525 \\
3 & 6.1714 & 0.4033 & 0.1664 & 0.1218 & 0.1664 & 0.0722 & 0.0252 & 0.0343 & 1.24 & 0.0277 \\
\hline
\end{tabular}

As shown in Table 2, it can be seen that judgment matrices $B_{1}, B_{2}, B_{3}$ all meet the consistency testing requirement $\left(C R_{k}<0.1\right)$.

Step 4: Calculate the total weight of each parameter in the scheme layer and check the consistency of the evaluation system.

The calculation results of each parameter weight can be shown in Table 3.

Table 3. Weights of each parameter in the scheme layer.

\begin{tabular}{cc}
\hline Parameter & Weight of Each Parameter \\
\hline CF & $\omega_{1}=a_{1} b_{11}+a_{2} b_{21}+a_{3} b_{31}=0.1689$ \\
PW & $\omega_{2}=a_{1} b_{12}+a_{2} b_{22}+a_{3} b_{32}=0.1498$ \\
PRI & $\omega_{3}=a_{1} b_{13}+a_{2} b_{23}+a_{3} b_{33}=0.1746$ \\
SMR & $\omega_{4}=a_{1} b_{14}+a_{2} b_{24}+a_{3} b_{34}=0.3359$ \\
AOA & $\omega_{5}=a_{1} b_{15}+a_{2} b_{25}+a_{3} b_{35}=0.0378$ \\
PA & $\omega_{6}=a_{1} b_{16}+a_{2} b_{26}+a_{3} b_{36}=0.1429$ \\
\hline
\end{tabular}

The results of the consistency testing of hierarchical total sorting are as below.

$$
C R=\frac{a_{1} C I_{1}+a_{2} C I_{2}+a_{3} C I_{3}}{a_{1} R I_{1}+a_{2} R I_{2}+a_{3} R I_{3}}=0.0597<0.1
$$

Where, when $C R<0.1$, it denotes that the weights of each parameter in the scheme layer are reasonable and effective. As shown in Table 3, it can be seen that the order of weight from large to small is $\mathrm{SMR}>\mathrm{PRI}>\mathrm{CF}>\mathrm{PW}>\mathrm{PA}>\mathrm{AOA}$, and the SMR feature has the largest weight among these six characteristic parameters.

According to the comprehensive evaluation results above, a sketch map of using SMR in REID is shown in Figure 7. 


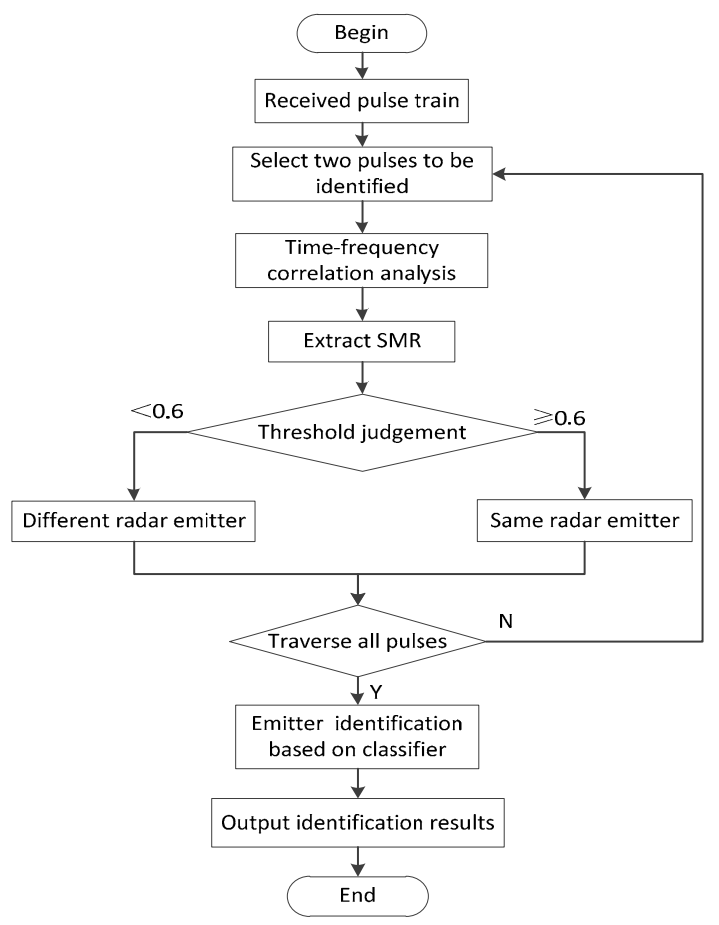

Figure 7. Sketch map of using SMR in radar emitter identification (REID).

\section{Simulation}

In this section, three experiments are performed to demonstrate the identification capability of the proposed coherent feature (SMR) based on the identification sketch map in Figure 7. The first experiment scene is different radar emitters with the same working mode; the second experiment scene is a certain radar emitter with complex working modes; the third experiment scene is different radar emitters with different working modes.

\subsection{Performance Evaluation of Experiment 1}

In this experiment, three radar emitters with the same working mode and the same modulation type are selected for validating the recognition capability of the SMR feature; the working mode is PRI staggering, the modulation type is $\mathrm{CW}$, and the three radar emitters are placed in three directions close to each other. Signal parameters are shown in Table 4.

Table 4. Simulation parameters.

\begin{tabular}{ccccccc}
\hline Emitter & PA & CF(MHz) & AOA & PW $(\boldsymbol{\mu s})$ & PRI $(\boldsymbol{\mu s})$ & Pulse Number \\
\hline Emitter 1 & 1 & 30 & $49.8^{\circ}$ & 5 & $46,50,54$ & 180 \\
Emitter 2 & 1 & 30 & $50^{\circ}$ & 5 & $46,50,54$ & 180 \\
Emitter 3 & 1 & 30 & $50.2^{\circ}$ & 5 & $46,50,54$ & 180 \\
\hline
\end{tabular}

If the typical five parameters and the intrapulse feature are utilized for emitter identification, it is easily misjudged that these pulses are from the same radar emitter. When SMR is combined with the existing five parameters for recognition, the accuracy of REID using SMR in different SNRs is shown in Figure 8. 


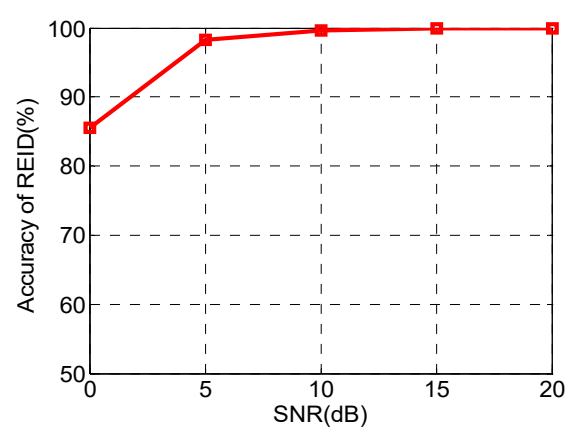

Figure 8. Accuracy of REID by using SMR in different SNRs.

From Figure 8, it can see that using SMR in REID can easily distinguish these three radar emitters and that the recognition accuracy has been improved.

\subsection{Performance Evaluation of Experiment 2}

In this experiment, a frequency-agile radar emitter is utilized to verify the recognition capability of the SMR feature. One hundred and eighty pulses were transmitted from the frequency-agile radar; the frequency values were 27, 32, and $37 \mathrm{MHz}$, Fs was $100 \mathrm{MHz}$. The other parameters of the frequency agile signal are shown in Table 5.

Table 5. Simulation parameters.

\begin{tabular}{cccccc}
\hline Pulse Train & PA & CF(MHz) & PW $(\boldsymbol{\mu s})$ & PRI $(\boldsymbol{\mu s})$ & Pulse Number \\
\hline Pulse train 1 & 1 & 27 & 10 & 90 & 60 \\
Pulse train 2 & 1 & 32 & 10 & 90 & 60 \\
Pulse train 3 & 1 & 37 & 10 & 90 & 60 \\
\hline
\end{tabular}

If the traditional five parameters are used for emitter identification, it also easily misjudges that the three pulse trains are from different radar emitters. The error probability of REID using SMR in different SNRs when SMR is combined with the existing five parameters for recognition is shown in Figure 9.

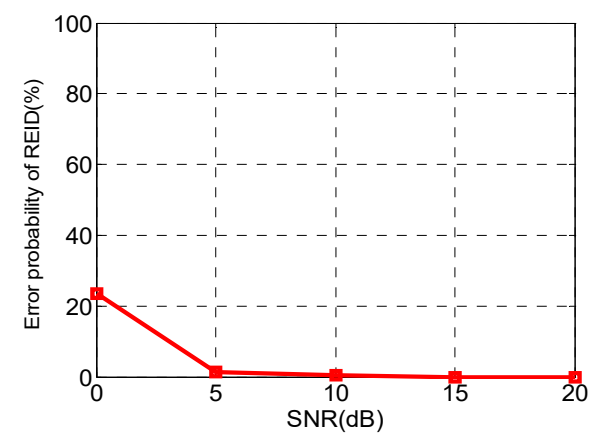

Figure 9. Error probability of REID by using SMR in different SNRs.

\subsection{Performance Evaluation of Experiment 3}

In this experiment, we demonstrate the recognition capability of the SMR feature on three different emitters with different working modes. The working mode of radar emitters 1-3 are, respectively, PRI staggering, frequency agility, and PRI jittering, and the three radar emitters are placed in three directions close to each other. All testing samples were from the emitter sample repositories. The other parameters are shown in Table 6. 
Table 6. Simulation parameters.

\begin{tabular}{ccccccc}
\hline Emitter & PA & CF(MHz) & AOA & PW $(\mu \mathbf{s})$ & PRI $(\mu \mathbf{s})$ & Pulse Number \\
\hline Emitter 1 & 1 & 27 & $49.8^{\circ}$ & 5 & $46,50,54$ & 200 \\
Emitter 2 & 1 & 27,33 & $50^{\circ}$ & 8 & 50 & 200 \\
Emitter 3 & 1 & 33 & $50.2^{\circ}$ & 5 & $50 \pm 5$ & 200 \\
\hline
\end{tabular}

Convolution neural network (CNN) and support vector machine (SVM) are taken for REID. The parameter features selected for REID are CF, PRI, PW, and SMR, which were freely combined to form one-dimensional, two-dimensional, three-dimensional, and four-dimensional feature vectors for the classifier.

In this experiment, the structure diagram of CNN [29] is shown in Figure 10.

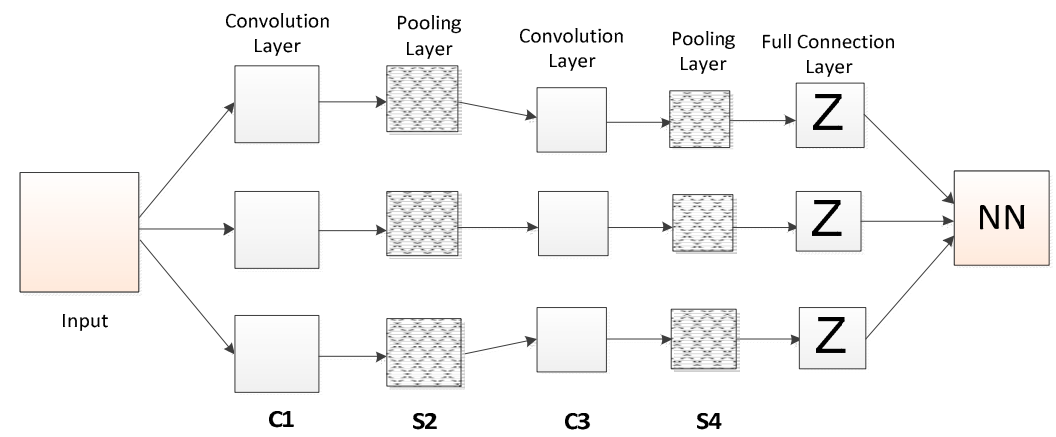

Figure 10. Structure diagram of convolution neural network (CNN).

In the structure of CNN, we select the optimized network structure "5C-2S-7C-2S-5-3" in terms of reference [30]. In this network structure, it denotes the convolution kernel size of the convolution layer (C1) is 5, the downsampling multiple of the pooling layer (S2) is 2, the convolution kernel size of the convolution layer (C3) is 7, the downsampling multiple of the pooling layer (S4) is 2, the number of training iterations is 5 , and the training data are divided into 3 segments. The training parameters of $\mathrm{CNN}$ are the learning rate is 0.01 , the weight decay is 0.0005 , and the momentum constant is 0.8 . All experiment data are composed of 600 samples containing three radar emitters from the simulation samples. Three hundred and sixty samples were utilized for training, and the other samples were used for testing. The recognition results based on $\mathrm{CNN}$ by different dimensional feature vectors in different SNRs are shown in Figure 11.

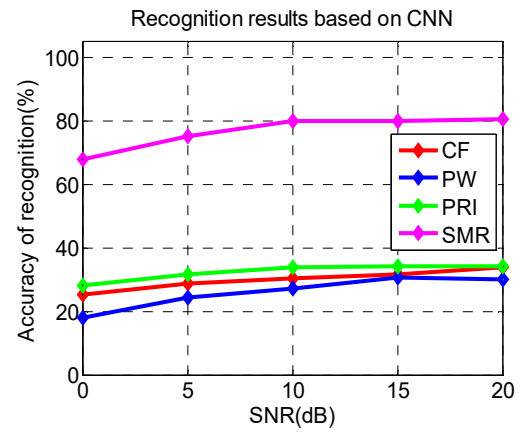

(a) One-dimensional feature vector.

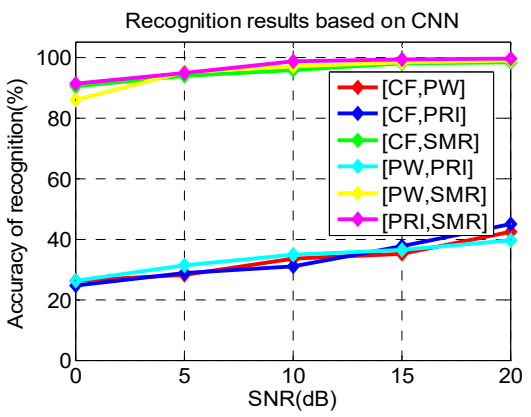

(b) Two-dimensional feature vector.

Figure 11. Cont. 


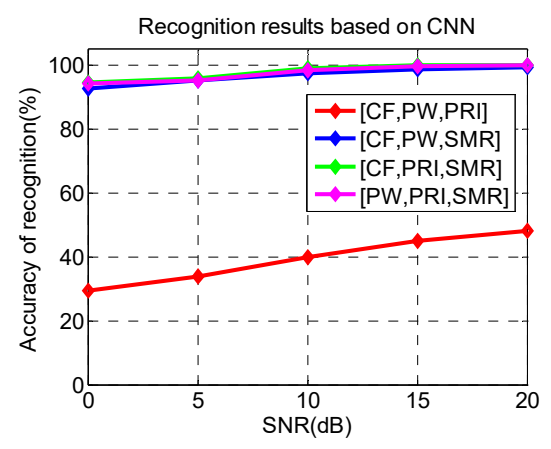

(c) Three-dimensional feature vector.

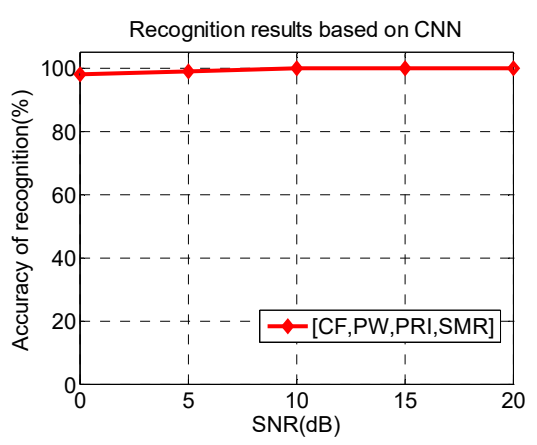

(d) Four-dimensional feature vector.

Figure 11. Recognition results of REID based on CNN in different SNRs.

In another SVM classifier [31], radial basis function (RBF) was selected as the kernel function, the parameter of the kernel function was $\gamma=0.1$, the penalty coefficient was $C=2$. The recognition results based on SVM by different dimensional feature vectors in different SNRs are shown in Figure 12.

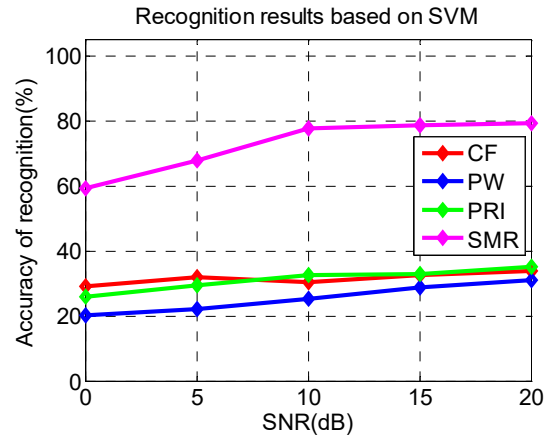

(a) One-dimensional feature vector.

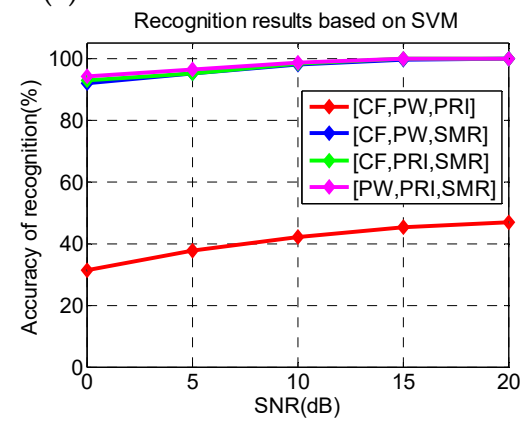

(c) Three-dimensional feature vector.

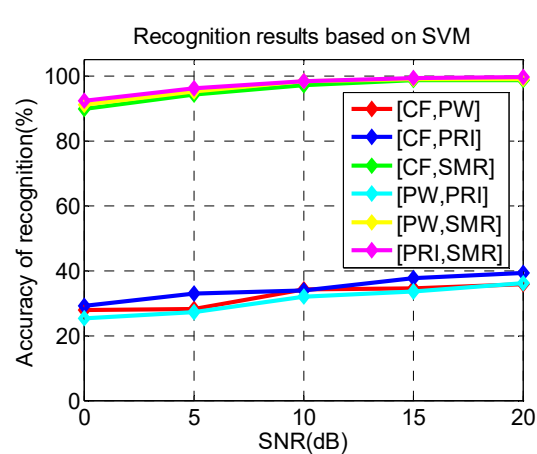

(b) Two-dimensional feature vector.

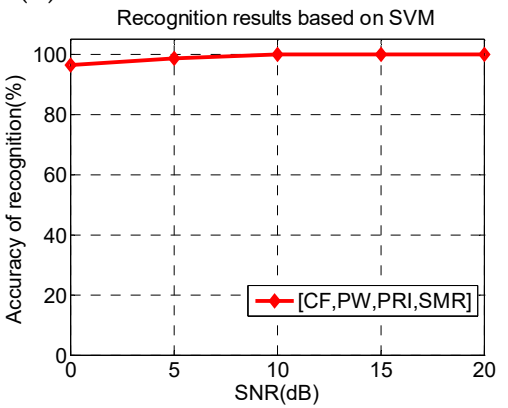

(d) Four-dimensional feature vector.

Figure 12. Recognition results of REID based on SVM in different SNRs.

As can be seen from Figures 11 and 12, when using the CNN and SVM classifiers, the recognition results are similar. In different SNRs, when a one-dimensional feature vector is utilized for recognition, the recognition accuracy based on the SMR feature is higher than other feature parameters. When the multidimensional feature vectors are used for recognition, if the feature vector contains the SMR feature, the recognition accuracy is also higher. It is further verified that the SMR feature has a better recognition ability. Furthermore, because of the self-training process of $\mathrm{CNN}$, when we have a emitter sample database, we should choose the CNN classifier first. However, when there is no sample database, the SVM classifier is preferred.

\section{Conclusions}

Aiming at the problem of reliability degradation of REID based on the typical five parameters in a complex electromagnetic environment, a new feature parameter based on the instantaneous 
autocorrelation function has been extracted, which makes full use of the coherent characteristics in today's coherent radar. Through simulation analysis, it has been validated that the proposed feature is insensitive to SNR and obtains strong adaptability in signal modulation change. Simulation results show that the coherent feature can not only achieve satisfactory identification results when combined with the typical five parameters, but it can be used as a new feature parameter to make up the shortcomings in today's radar emitter identification. Further work will focus on applying the proposed feature to REID in actual electromagnetic environments, and further validating the recognition capability of the proposed feature through practically measured data.

Author Contributions: Conceptualization, J.X. and L.J.; methodology, J.X. and X.Z.; software, J.X.; validation, J.X., L.T. and X.Z.; formal analysis, X.Z.; investigation, J.X.; resources, J.X.; data curation, L.T.; writing-original draft preparation, J.X.; writing-review and editing, X.Z.; visualization, L.T.; supervision, L.J.; project administration, X.Z.; funding acquisition, X.Z. All authors have read and agreed to the published version of the manuscript.

Funding: This research was funded in part by the National Nature Science Foundation of China (grant number 61976113).

Conflicts of Interest: The authors declare no conflict of interest.

\section{References}

1. Satija, U.; Trivedi, N.; Biswal, G.; Ramkumar, B.; Ramkumar, B. Specific Emitter Identification Based on Variational Mode Decomposition and Spectral Features in Single Hop and Relaying Scenarios. IEEE Trans. Inf. Forensics Secur. 2018, 14, 581-591. [CrossRef]

2. Ding, L.; Wang, S.; Wang, F.; Zhang, W. Specific Emitter Identification via Convolutional Neural Networks. IEEE Commun. Lett. 2018, 22, 2591-2594. [CrossRef]

3. Yuan, Y.; Cui, G.L.; Ge, M.M.; Yu, X.X. Active repeat jamming suppression via multi-static radar elliptic-hyperbolic location. In Proceedings of the 2017 IEEE Radar Conference, Seattle, WA, USA, 8-12 May 2017; Institute of Electrical and Electronics Engineers Inc.: Seattle, WA, USA, 2017; pp. 0692-0697.

4. Wu, X.; Hou, X.; Huang, Q.L.; Bu, Q. Radar Emitter Identification Algorithm Based on Fuzzy Set Theory. Command. Inf. Syst. Technol. 2018, 9, 55-59.

5. Huo, Q.; Lee, C.-H. A Bayesian predictive classification approach to robust speech recognition. IEEE Trans. Speech Audio Process. 2000, 8, 200-204. [CrossRef]

6. Zhang, B.; Srihari, S.N. Fast k-neatest neighbor classification using cluster-based trees. IEEE Trans. Pattern Anal. Mach. Intell. 2014, 26, 525-528. [CrossRef] [PubMed]

7. Yang, C.Z.; Wu, H.C.; Li, P.; Wang, M.L. Approach Based on Cloud Model and SVM for Emitter Identification. Modern Radar. 2013, 10, 41-45.

8. Xu, X. Radar Radiating-source Recognizing Based on Neural Networks. Comput. Digit. Eng. 2017, 45, 2126-2130.

9. Huang, Y.K.; Jin, W.D.; Yu, Z.B.; Wu, Y.P. Radar emitter signal recognition based on deep learning and ensemble learning. Syst. Eng. Electron. 2018, 40, 2420-2425.

10. Hong, S.; Kwak, S.; Han, B. Weakly Supervised Learning with Deep Convolutional Neural Networks for Semantic Segmentation: Understanding Semantic Layout of Images with Minimum Human Supervision. IEEE Signal Process. Mag. 2017, 34, 39-49. [CrossRef]

11. Li, Y.J.; Zhao, Y.J.; Zhao, C. Recognition of Intra-Pulse Feature of Radar Signals Based on Canonical Correlation Analysis. J. Inf. Eng. Univ. 2018, 19, 47-51.

12. Wang, H.; Fan, X.; Chen, Y.; Yang, Y. Wigner-Hough transform based on slice's entropy and its application to multi-LFM signal detection. J. Syst. Eng. Electron. 2017, 28, 634-642.

13. Kishore, T.R.; Rao, K.D. Automatic Intra Pulse Modulation Classification of Advanced LPI Radar Waveforms. IEEE Trans. Aerosp. Electron. Syst. 2017, 53, 901-914. [CrossRef]

14. Guan, X.; Zhu, H.P.; Zhang, Y.H. Analysis and Extraction of Radar Emitter Signal Feature based on Fractional Fourier Transform. J. Detect. Control 2018, 40, 71-76.

15. Yu, Z.B.; Jin, W.D.; Chen, C.X. Radar Emitter Signal Recognition Based on WRFCCF. J. Southwest Jiao Tong Univ. 2010, 45, 290-295. 
16. Yuan, Y.; Huang, Z.; Wu, H.; Wang, X. Special emitter identification based on Hilbert-Huang transform based time-frequency-energy distribution features. IET Commun. 2014, 8, 2404-2412. [CrossRef]

17. Guo, L.R.; He, M.H.; Yu, C.L.; Wang, B.Q. Describe of Frequency Agility Radar Signal Coherent Characteristic. Fire Control Command Control 2015, 40, 24-27.

18. Guo, L.R.; He, M.H.; Yu, C.L.; Wang, B.Q. New Time Domain Coherent Method of Radar Emitter Recognition. Mod. Def. Technol. 2015, 43, 108-113.

19. Han, L.H.; Huang, G.M.; Wang, P. Research into Signals Intra-pulse Analysis of Linear Frequency Modulation Radar Based on Instantaneous Auto-correlation Algorithm. Shipboard Electron. Countermeas. 2011, 34, 1-5.

20. Chen, L.J.; Shen, Y.L.; Chen, B.X.; Liu, P.; Chen, P. Frequency Estimation Algorithm Based on Multi-segment Auto Correlation Signal and MC Method. Instrum. Tech. Sens. 2016, 5, 92-94.

21. Chen, P.; Tu, Y.Q.; Li, M.; Liu, P. Frequency estimation method of data extension based on autocorrelation. Transducer Microsyst. Technol. 2019, 38, 26-29.

22. Ren, D.F.; Zhang, T.; Han, J. Specific emitter identification based on ITD and texture analysis. J. Commun. 2017, 38, 160-168.

23. Zhang, Y.; Hong, R.-J.; Pan, P.-P.; Deng, Z.-M.; Liu, Q.-F. Frequency-Domain Range Sidelobe Correction in Stretch Processing for Wideband LFM Radars. IEEE Trans. Aerosp. Electron. Syst. 2017, 53, 111-121. [CrossRef]

24. Lu, W.; Xie, J.; Wang, H.; Sheng, C. Parameterized time-frequency analysis to separate multi-radar signals. J. Syst. Eng. Electron. 2017, 28, 493-502.

25. Xu, J.; He, M.H.; Han, J. Effectiveness assessment of radar emitter signal recognition based on AHP-interval-TOPSIS. J. Air Force Early Warn. Acad. 2014, 28, 243-246.

26. Fan, X.; Fang, Y.; Cheng, Z.; Zhu, B.; Shi, Z. Performance Evaluation for Tracking Algorithm Based on AHP. J. Proj. Rockets Missiles Guid. 2013, 33, 101-105.

27. Zhu, B.; Jin, W.D.; Yu, Z.B. Feature evaluation for advanced radar emitter signals based on SPA-FAHP. J. Comput. Appl. 2014, 34, 1834-1838.

28. Long, F.; Liu, J.; Yu, H.; Zhu, H.; Zhang, S. The Evaluation System and Application of the Homestay Agglomeration Location Selection. J. Resour. Ecol. 2019, 10, 324-334.

29. Jin, Q.; Wang, H.Y.; Yan, M. Radar Emitter Signal Recognition Based on Deep Convolutional Network. Modern Deference Technol. 2019, 47, 154-160.

30. Leng, P.F.; Xu, C.Y. Specific Emitter Identification Based on Deep Reinforcement Learning. Acta Armamentarii 2018, 39, 2420-2426.

31. Vanhoy, G.; Schucker, T.; Bose, T. Classification of LPI radar signals using spectral correlation and support vector machines. Analog. Integr. Circuits Signal Process. 2017, 91, 305-313. [CrossRef] 